\title{
Uterine Polyp
}

National Cancer Institute

\section{Source}

National Cancer Institute. Uterine Polyp. NCI Thesaurus. Code C3662.

A benign protruding lesion arising either from the endometrial cavity (endometrial polyp) or the endocervix (endocervical polyp). It may occasionally recur following complete resection. 